\title{
Tuberculosis: are we making it incurable?
}

\author{
José A. Caminero ${ }^{1,2}$, Alberto Matteelli ${ }^{3}$ and Robert Loddenkemper ${ }^{4}$ \\ Affiliations: 'Dept of Pneumology, Universitary Hospital of Gran Canaria “Dr. Negrín”, Las Palmas de Gran \\ Canaria, Spain. International Union Against Tuberculosis and Lung Disease (The Union), Paris, France. \\ ${ }^{3}$ Institute of Infectious and Tropical Diseases, University of Brescia, Brescia, Italy. ${ }^{4}$ German Central Committee \\ against Tuberculosis (DZK), Berlin, Germany.
}

Correspondence: J.A. Caminero, Dept of Pneumology, Universitary Hospital of Gran Canaria "Dr. Negrín", Barranco de la Ballena s/n, 35010 Las Palmas de Gran Canaria, Spain. E-mail: jcamlundgobiernodecanarias.org

O @ERSpublications

With proper management all TB patients have a chance to be cured; it should never be thought that a TB case is incurable http://ow.ly/ksm5g

Tuberculosis (TB) is most probably the disease that has caused more damage to the human species throughout its history in terms of number of patients and, above all, death toll. There have been thousands of years spent fighting against Mycobacterium tuberculosis in which the human species could only rely on the efficiency of the immune system [1]. We must recall its importance as, in the pre-antibiotic era, the immune system alone could ensure important achievements such as: 1) only 50\% of the people exposed to $M$. tuberculosis contract the infection [2]);2) only $10 \%$ of those infected progress to active disease; and 3) up to $30 \%$ of patients with advanced TB disease heal spontaneously (fig. 1) [3, 4]. In spite of this, the fate of TB patients in the pre-chemotherapy period was very bleak, with a mortality of $>50 \%$ within 5 years after the onset of disease [3,4]. For centuries, several empirical treatments were attempted to trying to change this fateful prognosis. Additionally, sanatoriums were built throughout the world for many decades (fig. 2). However, all these interventions were not really effective, and none of them contributed significantly to improve the doom of TB patients [4]. Then, fortunately, their fate changed dramatically with the advent of the antibiotic era. The two decades from the discovery of streptomycin in 1943 to that of rifampicin in 1963 changed a devastating disease into a relatively easy to cure one. Not only were up to 11 different drugs with activity against $M$. tuberculosis discovered (streptomycin, p-aminosalicylate (PAS), thiacetazone, isoniazid $(\mathrm{H})$, pyrazinamide, cycloserine, kanamycin, ethionamide, ethambutol, capreomycin and rifampicin $(\mathrm{R})$ ), but, based on multiple randomised clinical trials, the basic fundamentals of TB treatment were also established $[4,5]$. Probably the most essential of these was the need to combine at least two to three drugs to which the patient was sensitive, as $M$. tuberculosis can develop resistance to single drugs.

The ability of M. tuberculosis to develop resistances to antibiotics was described in the very first randomised clinical trial conducted with streptomycin and published in 1948 [6]. The first great lesson learnt from early randomised clinical trials was that using TB drugs in monotherapy, or in poor combinations, would lead to the selection of resistance to these drugs [5]. Thus, as a result of the misuse of the available drugs, monoresistant TB started to develop, followed by poly-resistant TB (resistance to two or more drugs), multi-drug resistant (MDR)-TB (resistance to at least $\mathrm{H}+\mathrm{R}$ ), and extensively-drug resistant (XDR)-TB (defined as MDR-TB plus resistance to at least one fluoroquinolone (FQ) and one second-line injectable drug). The need for definitions beyond XDR-TB is a currently an issue of debate.

The definitions in the field of anti-TB drug resistance should be based on two main factors: 1 ) the capacity to reliably test anti-TB drugs in the laboratory, and 2) the possible different prognosis that a new definition could bear. As is well known, rifampicin is currently by far the best available drug, and is the only one that combines excellent bactericidal, sterilising and resistance preventing activity [7]. Therefore, it is the only drug with the ability to reduce the duration of TB treatment to 9 months (6 months if the other highly

Received: Dec 242012 | Accepted after revision: Jan 292013

Conflict of interest: None declared.

Copyright (C)ER 2013 


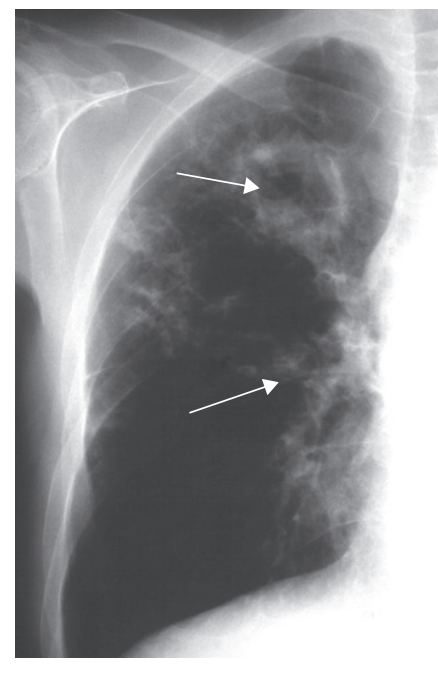

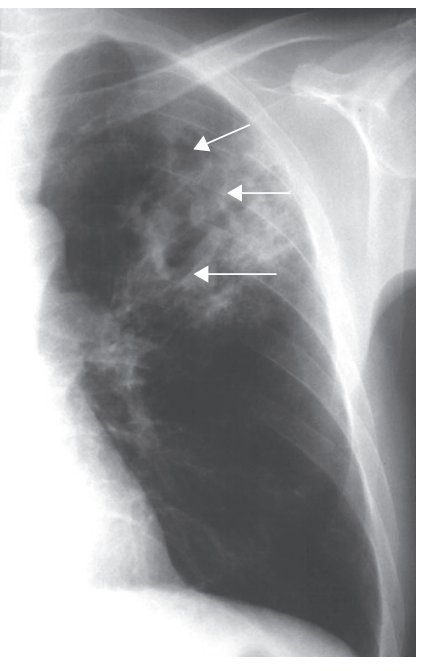

FIGURE 1 Chest radiograph showing extensive cavitary tuberculosis (arrows).

sterilising drug, pyrazinamide $(Z)$ is included) [5]. The lack of $R$ in a regimen not only requires the extension of the treatment duration to a minimum of 12-18 months, but also clearly worsens the prognosis of the patient [4]. In addition, the clinical reliability of drug susceptibility testing for $\mathrm{R}$ is very high. Thus, resistance to $\mathrm{R}$ is the basis of the currently accepted definition of MDR-TB, although, little by little, more people are supporting the view that even mono- or poly-resistance to $\mathrm{R}$ with in vitro susceptibility to $\mathrm{H}$ (which is very uncommon) should be accepted as the definition of MDR-TB. The achievable treatment success rate for MDR-TB cases ranges between $62 \%$ and $69 \%$ using long and poorly tolerated second-line drug regimens $[8,9]$. These success rates can be achieved despite the absence of $\mathrm{R}$ and $\mathrm{H}$ in the regimen because we have two other families of drugs with high efficacy against M. tuberculosis: the FQs and the second-line drug injectables (kanamycin, amikacin and capreomycin) [7], both with reproducible and relatively reliable drug susceptibility tests. Therefore, the definition of XDR-TB covers these two families of drugs, which can be tested in the laboratory and whose resistance carries a clearly worse prognosis, with a success rate of just $43.7 \%$ as reported in the meta-analysis by JACOBSON et al. [10]. There is convincing evidence that FQs are associated with a better prognosis when used for the treatment of MDR-TB [9, 11, 12]. Interestingly, the meta-analysis carried out by JACOBSON et al. [10] showed that a success rate of $59 \%$ could be reached in those settings where a later generation FQ was systematically used to treat XDR-TB strains which, by definition, are resistant to FQs.

This issue of the European Respiratory Journal contains two important articles that shed light on the diversity of the prognosis of patients with strains with MDR-TB and beyond. The first article by FALzON et al. [13] analyses the outcome of patients with a gradient of resistance from MDR-TB (without additional resistances) to XDR-TB. In the second article MigLIORI et al. [14] discuss the appropriateness of adopting a

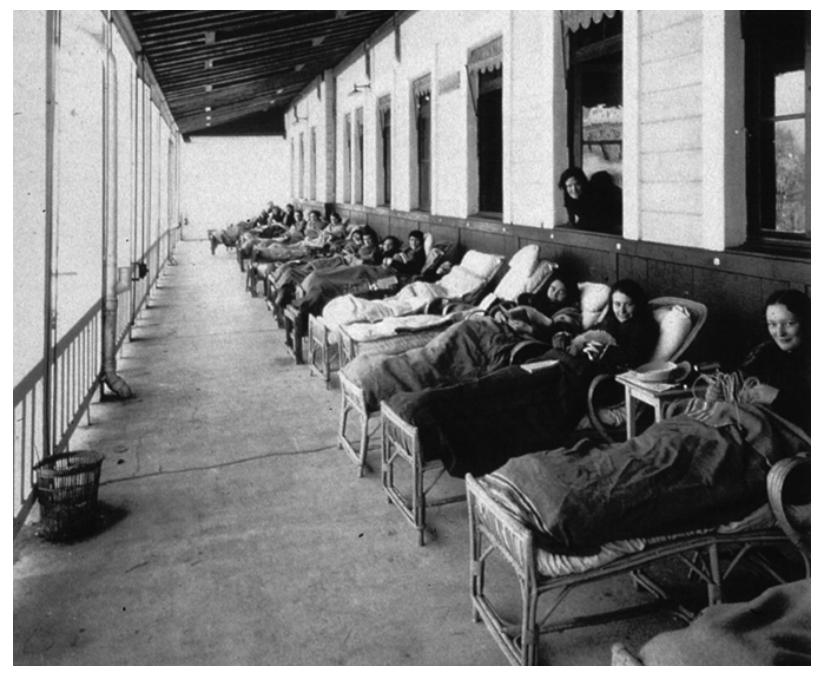

FIGURE 2 A photograph showing a sanatorium, which were built with large patios where tuberculosis patients could rest. 
definition beyond XDR-TB, based on the worse prognosis found when the resistance pattern extends, especially to all second-line drug injectables.

The most important finding in the article by FALzON et al. [13] is that, among the two drug families that constitute the treatment backbone for patients with MDR-TB, FQs, especially the later generation ones, are better than second-line drug injectables. The treatment success rate (compared to treatment failure, relapse and death) among almost $7000 \mathrm{MDR}-\mathrm{TB}$ patients from 26 experienced centres was progressively decreasing from $64 \%$ for patients with MDR-TB without additional resistance, to 56\% for MDR-TB with additional resistance to second-line drug injectables only, to $48 \%$ for MDR-TB with additional resistance to FQs only, and to $40 \%$ for XDR-TB patients. Remarkably, this poor outcome of $40 \%$ for XDR-TB patients is very close to the $43 \%$ published by JACOBSON et al. [10].

The findings of FALzON et al. [13] suggest that the XDR-TB definition adopted in 2006 [15] may not be the most appropriate one, because, for instance, patients with MDR-TB with resistance to ofloxacin and kanamycin alone (by definition XDR-TB cases, although the strain can be susceptible to all other first-line drugs, to a later generation FQ and to other second-line drug injectables such as capreomycin) have a prognosis which is closer to that of MDR-TB than XDR-TB [16]. Following this reasoning, the correct definition of XDR-TB should probably cover all first-line drugs (not only $\mathrm{H}$ and R), plus all FQs and all second-line drug injectables [17].

The data published by MigLiori et al. [14] in this issue support this hypothesis. Their data show that among patients with XDR-TB (405 in total), the poor prognosis of those without further resistance $(n=301$, treatment success $43 \%)$ is worsened by the addition of resistance to all second-line drug injectables $(n=68$, treatment success 34\%), and made worse further by the addition of resistance to ethambutol and/or pyrazinamide $(n=42$, treatment success $19 \%)$. Interestingly, prognosis was not affected by resistance to any of the group 4 drugs (ethionamide/prothionamide, cycloserine/terizidone or PAS) in the presence of resistance to all second-line drug injectables. These results are slightly in contrast to the published findings of a recent meta-analysis including the largest number of MDR-TB patients published to date, in which, in addition to FQs, the possibility of using ethionamide/prothionamide was associated with a better profognosis [18].

The article by FALzON et al. [13] provides additional important original information. First, it reports that, contrary to the case of MDR-TB which requires an ideal number of four drugs for effective treatment [19, 20 ], in the case of XDR-TB the use of at least six drugs in the intensive phase is associated with a better treatment success. Secondly, it suggests that the duration of treatment recommended for MDR-TB is also appropriate to treat XDR-TB [19].

The conclusions reached by MigLiori et al. [14] are important. The authors admit that, in spite of the evidence that the current XDR-TB definition may not be the best to predict the outcome of affected patients, limitations in availability, accuracy and reproducibility of current drug susceptibility methods preclude the adoption of a useful definition beyond the one currently used for XDR-TB. We agree with this statement, although, perhaps, the current definition of XDR-TB could be revised in order to include all first-line oral drugs (MDR-TB+ethambutol+pyrazinamide), all FQs and all second-line drug injectables [17].

In summary, it seems that the chance of cure for patients with XDR-TB plus resistance to all second-line drug injectables is practically the same as for patients with cavitary TB not receiving any treatment in the pre-antibiotic era [3]. Moreover, the chance of cure for patients with XDR-TB without widespread resistance (treatment success $40-43 \%$ ) is slightly better than the spontaneous healing produced by the action of our immune system. It is of interest to note that, in order to reach a relatively poor treatment outcome, very lengthy toxic and expensive regimens have to be used. The low cure rate for XDR-TB is surprising, in light of the evidence from the pre-rifampicin and pre-FQ studies, as most TB patients with resistance to streptomycin plus PAS plus isoniazid (who were very similar to current XDR-TB cases) were cured when combining only three drugs to which the patient was sensitive [21-24]. The reasons for such discrepancies are unclear: the older findings were all derived from a very individualised management in selective referral centres, and indirect evidence that treatment of $\mathrm{TB}$, even in patients with a very extensive pattern of resistance (beyond XDR-TB), is positively affected by the adoption of the best available standard of care. The high chances of cure recently published for patients with XDR-TB support this view [25]. Still, it is worrisome and worth noting that the clinical centres contributing cases to the series analysed by both FALzon et al. [13] and MigLiori et al. [14] represent centres of excellence: a warning not to surrender to complacency when dealing with XDR-TB.

Many mistakes in the use of anti-TB drugs have led to the current situation with an increasing number of $\mathrm{TB}$ patients carrying strains with a very extensive pattern of resistance. What is even worse is that patients with $\mathrm{TB}$ with widespread resistance still suffer many clinical (e.g. addition of only one to two drugs to a 
failing regimen, or irrational use of available effective anti-TB drugs) or programmatic (no social support, no good approach to potential adverse drug reactions, no good adherence supporting initiatives, etc.) errors $[26,27]$. If this trend is not reversed, the chances of cure for TB patients will remain low, and the high costs of treating MDR- or XDR-TB may become unaffordable in low-income countries [28].

One final remark, even considering the clear evidence that the prognosis of TB patients worsens when the pattern of resistance increases, it should never be accepted that a TB case is incurable. All TB patients, even those with XDR-TB or beyond XDR-TB, should always have a chance to be treated using the available clinical and programmatic management strategies. The arrival of new drugs [29] will certainly enhance our capacity to cure TB patient, if we are able to protect them from the ability of M. tuberculosis to generate resistance $[19,20]$, and will ensure their durability to a time range similar to the very long one elapsing from the discovery of a new compound to its registration for the market use.

\section{References}

Caminero JA, Torres A. Controversial topics in tuberculosis. Eur Respir J 2004; 24: 895.

Stead WW. Variation in vulnerability to tuberculosis in America today: random, or legacies of different ancestral epidemics? Int J Tuberc Lung Dis 2001; 5: 807-814.

3 Grzybowski S, Enarson DA. The fate of cases of pulmonary tuberculosis under various treatment programmes. Bull Int Union Tuberc Lung Dis 1978; 53: 70-75.

4 Caminero JA. A tuberculosis guide for specialist physicians. Paris, International Union Against Tuberculosis and Lung Disease, 2003.

5 Fox W, Ellard GA, Mitchison DA. Studies on the treatment of tuberculosis undertaken by the British Medical Research Council tuberculosis units, 1946-1986, with relevant subsequent publications. Int J Tuberc Lung Dis 1999; 3: Suppl. 2, S231-S279.

Streptomycin treatment of pulmonary tuberculosis. BMJ 1948; 2: 769-783.

7 Caminero JA, Sotgiu G, Zumla A, et al. Best drug treatment for multidrug-resistant and extensively drug-resistant tuberculosis. Lancet Infect Dis 2010; 10: 621-629.

8 Orenstein EW, Basu S, Shah NS, et al. Treatment outcome among patients with multidrug-resistant tuberculosis: systematic review and meta-analysis. Lancet Infect Dis 2009; 9: 153-161.

9 Johnston JC, Shahidi NC, Sadatsafavi M, et al. Treatment outcomes of multidrug-resistant tuberculosis: a systematic review and meta-analysis. PLoS One 2009; 4: e6914.

10 Jacobson KR, Tierney DB, Jeon CY, et al. Treatment outcomes among patients with extensively drug-resistant tuberculosis: systematic review and meta-analysis. Clin Infect Dis 2010; 51: 6-14.

11 Chan ED, Laurel V, Strand MJ, et al. Treatment and outcome analysis of 205 patients with multidrug-resistant tuberculosis. Am J Respir Crit Care Med 2004; 169: 1103-1109.

12 Chiang CY, Enarson DA, Yu MC, et al. Outcome of pulmonary multidrug-resistant tuberculosis: a 6-year follow-up study. Eur Respir J 2006; 28: 980-985.

13 Falzon D, Gandhi N, Migliori GB, et al. Resistance to fluoroquinolones and second-line injectable drugs: impact on multidrug-resistant TB outcomes. Eur Respir J 2013; 42: 156-168.

14 Migliori GB, Sotgiu G, Gandhi NR, et al. Drug resistance beyond extensively drug-resistant tuberculosis: individual patient data meta-analysis. Eur Respir J 2013; 42: 169-179.

15 World Health Organization, Stop TB Partnership. The global MDR-TB \& XDR-TB response plan 2007-2008. Geneva, World Health Organization, 2007.

16 Migliori GB, Besozzi G, Girardi E, et al. Clinical and operational value of the extensively drug-resistant tuberculosis definition. Eur Respir J 2007; 30: 623-626.

17 Caminero JA. Extensively drug-resistant tuberculosis: is its definition correct? Eur Respir J 2008; 32: 1413-1415.

18 Ahuja SD, Ashkin D, Avendano M, et al. Multidrug resistant pulmonary tuberculosis treatment regimens and patient outcomes: an individual patient data meta-analysis of 9,153 patients. PloS Med 2012; 9: e1001300.

19 Falzon D, Jaramillo E, Schünemann HJ, et al. WHO guidelines for the programmatic management of drug-resistant tuberculosis: 2011 update. Eur Respir J 2011; 38: 516-528.

20 World Health Organization. Guidelines for the programmatic management of drug-resistant tuberculosis. Emergency update 2008. Geneva, World Health Organization, 2008.

21 Caminero JA. Treatment of multidrug-resistant tuberculosis: evidence and controversies. Int J Tuberc Lung Dis 2006; 10: 829-837.

22 Zierski M, Zachara A. Late results in re-treatment of patients with pulmonary tuberculosis. Tubercle 1970; 51 : $172-177$.

23 Tousek J, Jancik E, Zelenka M, et al. The results of treatment in patients with cultures resistant to streptomycin, isoniazid and PAS: a five-year follow-up. Tubercle 1967; 48: 27-31.

24 Pines A. The fate of patients with drug-resistant tubercle bacilli and pulmonary tuberculosis. Br J Dis Chest 1962; 56: $163-170$

25 Mitnick CD, Shin SS, Seung KJ, et al. Comprehensive treatment of extensively drug-resistant tuberculosis. $N$ Engl J Med 2008; 359: 563-574.

26 Migliori GB, Sotgiu G, D’Ambrosio L, et al. TB and MDR/XDR-TB in European Union and European Economic Area countries: managed or mismanaged? Eur Respir J 2012; 39: 619-625.

27 Sotgiu G, D'Ambrosio L, Centis R, et al. TB and M/XDR-TB infection control in European reference centres: the Achilles' heel? Eur Respir J 2011; 38: 1221-1223.

28 Loddenkemper R, Sotgiu G, Mitnick CD. Cost of tuberculosis in the era of multidrug resistance: will it become unaffordable? Eur Respir J 2012; 40: 9-11.

29 Grosset JH, Singer TG, Bishai WR. New drugs for the treatment of tuberculosis: hope and reality. Int J Tuberc Lung Dis 2012; 16: 1005-1014. 